\title{
Par de là l'ethnicité : vers une citoyenneté culturelle Latina
}

Kristina Tiedje

\section{(2) OpenEdition \\ 12 Journals}

Édition électronique

URL : https://journals.openedition.org/pa/1908

DOI : 10.4000/pa.1908

ISSN : 2273-0362

Éditeur

Université Lumière Lyon 2

Édition imprimée

Date de publication : 1 janvier 2006

Pagination : 65-77

ISBN : 1643-7706

ISSN : 1634-7706

Référence électronique

Kristina Tiedje, «Par de là l'ethnicité : vers une citoyenneté culturelle Latina », Parcours

anthropologiques [En ligne], 6 | 2006, mis en ligne le 06 juillet 2021, consulté le 21 juillet 2021. URL :

http://journals.openedition.org/pa/1908; DOI : https://doi.org/10.4000/pa.1908 


\title{
Par de là l'ethnicité : VERS UNE CITOYENNETÉ CULTURELLE LATINA
}

Kristina Tiedje

Université Lyon 2

\begin{abstract}
"A contemporary "politics of citizenship" must take into account the role which the social movements have played in expanding claims to rights and entitlements to new areas. It must address not only issues of class and inequality, but also questions of membership posed by feminism, the black and ethnic movements, ecology (including the moral claims of animal species and of Nature itself) and vulnerable minorities. »
\end{abstract}

Stuart Hall et David Held (1990)

Les études sur les nouveaux mouvements sociaux ont élargi la notion de citoyenneté (jusqu'alors pensée à travers ses aspects politiques et légaux généraux) à des dimensions subjectives procédant de politiques culturelles et identitaires. Ainsi que l'écrit Renato Rosaldo (1994), la notion de " citoyenneté culturelle " implique des revendications culturelles de sujets historiquement exclus de la citoyenneté légale (qui tend à effacer les différences culturelles). Dans cette perspective, il s'agira ici, se basant sur des données ethnographiques et des récits de Latinos recueillis sur les champs agricoles dans l'Oregon, où travaillent des ouvriers d'origine mexicaine (en majorité zapotèques et mixtèques), de comprendre, pardelà l'ethnicité, l'émergence d'une " citoyenneté culturelle » latina - et ce, au moyen d'un questionnement en deux temps.

Nous nous demanderons d'abord quelles sont les politiques étatiques qui inscrivent des identités ethniques et influencent ainsi la construction des ethnicités par les sujets - question renvoyant à la dialectique «se faire une identité » ou « être produit » (Ong 1996)? D'un côté, les politiques de la citoyenneté visent à contrôler les citoyens à travers des institutions pour assurer la bonne gouvernance, de l'autre côté, les sujets s'engagent dans des processus de self-making afin de se construire leurs identités culturelles. La seconde question, liée à la précédente, abordera plus particulièrement la « citoyenneté culturelle » à travers les notions de droits et de communauté chez les Latinos d'origine mexicaine dans l'Oregon. On 
se demandera ainsi dans quelle mesure les revendications d'une appartenance culturelle à une communauté latina aux États-Unis contestent les désignations ethniques et raciales instaurées par les institutions de l'État en vue d'établir des critères d'une citoyenneté universelle.

Rappelons que les Latinos forment une communauté ethniquement et linguistiquement hétérogène. Pourtant, les immigrants récents sans carte de résidence, ainsi que les Latinos issus de l'immigration se mobilisent ensemble pour revendiquer l'appartenance à une communauté, à de nouveaux espaces publics et à des droits collectifs aux ÉtatsUnis. Fort de ce constat, nous apporterons d'abord des outils conceptuels pour comprendre la notion de « citoyenneté culturelle » en tant que pratique de subjectivation s'inscrivant dans une dialectique qui réunit politiques de l'État et expériences subjectives. Par la suite, nous interrogerons l'idée de racisation et d'ethnicisation des Latinos par le haut et au moyen de labels raciaux et ethniques propres aux politiques d'immigration. Enfin, les revendications culturelles et identitaires nous apparaîtront comme autant de processus subjectifs qui trouvent, dans leurs expériences communes du monde de travail, un dénominateur commun pour créer une communauté latina.

\section{LA CITOYENNETÉ CULTURELLE COMME PROCESSUS IDENTITAIRE}

La citoyenneté, dans sa conception légale est juridique, vise à fonder l'appartenance des sujets à l'État démocratique et à la société civile, ainsi qu'à définir leur positionnement au sein de l'État-nation, en dépit des différences culturelles et ethniques. Ainsi, même si la citoyenneté n'est pas une catégorie universelle mais une idée, comme nous le savons, toute subjective, les politiques étatiques continuent de proposer un modèle universel de citoyenneté, modèle qui semble pourtant en décalage avec, aux États-Unis par exemple, les revendications des Latinos ou, plus généralement, avec les politiques identitaires de sujets aux origines ethniques et culturelles diverses dans ce grand pays.

Pour Aihwa Ong (1996 : 737), les immigrés aux États-Unis se trouvent face à un État qui les contraint à être des sujets d'un État-nation. Ong considère que la citoyenneté culturelle revient ici à un processus de subjectivation (au sens foucaldien du terme) : un processus où le sujet se construit tout en étant construit « par les relations de pouvoir qui produisent un consentement par les schèmes de surveillance, de discipline, de contrôle, et d'administration » (Foucault, 1989, cité dans Ong, ibid.). Dans le contexte des démocraties occidentales, et en particulier dans le contexte nord-américain, Philip Corrigan et Derek Sayer soulignent que les politiques de l'État visent à " universaliser la notion de citoyenneté » pour donner un aspect homogène et unifié aux expressions diverses de la réalité des groupes qui vivent au sein de son territoire (cité dans Ong, 1996 : 738). La « citoyenneté universelle » relève d'un processus d'individuation qui " construit » le sujet dans des catégories socioprofessionnelles : ouvriers, payeurs d'impôts ou chômeurs, par exemple. C'est le 
monde du travail qui crée une expérience partagée par tous les citoyens et qui définit leurs liens avec les institutions de l'État.

Nous retiendrons ici que la citoyenneté culturelle est un processus double : la citoyenneté universelle, promue par l'État, suscite a contrario des attitudes et des expériences subjectives d'individus (citoyens ou non) qui négocient leur statut au sein des États-Unis. Ong (1996) appuie ce constat quand, pour elle, la citoyenneté culturelle ne saurait être un processus unilatéral qui permettrait aux immigrants " sans papiers » et aux résidents issus de l'immigration de mettre en avant une identité commune, telle que la propose Renato Rosaldo (1994, 1997). De fait, les groupes qui commencent à revendiquer une identité latina transforment les catégories racisantes, imposées par les politiques de l'État, en des catégories permettant une construction identitaire et communautaire. Il s'agit donc, pour nous, de considérer les expériences communes de ces divers types de population en fonction de paramètres culturels, économiques, historiques et sociaux, qui composent les pratiques de stigmatisation et de subjectivation des Latinos aux États-Unis.

\section{L'HÉTÉROgÉNÉITÉ des LATINOS dES ÉtATS-UNIS}

Depuis les années 1980, le pourcentage de Latinos aux États-Unis augmente à une vitesse imprévue, phénomène relevant d'une véritable " latinisation » démographique des États-Unis. Cela s'explique par la globalisation et le libéralisme économiques qui ont depuis longtemps influencé les relations et les flux migratoires entre les États-Unis et l'Amérique latine. En 1980, il y avait environ quatorze millions de Latinos aux États-Unis. Vingt ans après, en 2000, leur nombre est de plus de trentecinq millions, la plupart d'entre eux étant de nouveaux migrants. Selon des calculs récents, les Latinos seront la "première minorité » du pays d'ici 2050, puisqu'alors plus nombreux que toutes les autres minorités réunies (Cohen, 2004; Delgado \& Stefancic, 1998). Remarquons que, dans ces conditions, la notion de «minorité » perd tout son sens. De plus, les Latinos des États-Unis forment un groupe très hétérogène et le flux d'immigration de ces dernières décennies accentue cette diversification (Cohen \& Tréguer, 2004). La majorité des Latinos étant celle d'immigrants ou d'enfants d'immigrants, il n'est donc pas surprenant que les expériences subjectives des Latinos aux USA soient liées aux politiques de citoyenneté.

On s'aperçoit, en outre, que les expériences des Latinos sont variées si l'on examine les différents groupes installés aux États-Unis : les Mexicains, les Porto-Ricains, les Cubains, les Dominicains et ceux provenant d'Amérique centrale ou d'Amérique du Sud. L'expérience des Latinos diffère aussi selon le lieu où ils résident. Il est alors difficile de généraliser le cas des Latinos en Floride, à Chicago, à New York, dans les grandes villes en général, ou dans les zones rurales, dans l'Oregon, au Texas, en Californie. Par conséquent, l'argument fréquent soutenant que les Latinos font partie d'une grande famille ou d'un collectif homogène risque 
de poser un problème (Delgado \& Stefancic, 1998 : XVII) - surtout si l'on considère les contradictions, les tensions et les conflits, autrement dit, les fissures qui se produisent autour des expériences subjectives liées aux catégories de classe, de " race ", catégorie culturelle et sociale admise par beaucoup, et de couleur. Cette hétérogénéité de fait nous invite à nous méfier de tout essentialisme latino. Or, on peut observer l'émergence de revendications d'une « citoyenneté culturelle latina ».

\section{LeS PHÉNOMÈNES DE RACISATION ET D'ETHNICISATION}

On ne peut considérer l'émergence des revendications d'une citoyenneté culturelle latina comme un processus de subjectivation sans interroger la continuité entre le passé colonial et les politiques contemporaines de racisation des Latinos - perspective déjà tracée par Lars Schoultz (1998). L'auteur a, en effet, étudié deux cents ans de l'histoire des politiques américaines envers l'Amérique latine, révélant que l'élite nordaméricaine aurait, tout ce temps, construit l'idée d'une infériorité raciale des Latino-américains. Pour mettre à jour ces préjugés racistes, Schoultz cite Joel Poinsett, un ambassadeur américain au Mexique au XIX siècle qui aurait affirmé : «Les Mexicains sont une race ignorante et immorale et ont des relations constantes avec les aborigènes, qui étaient et sont toujours dégradés à la classe la plus basse des êtres humains. » (Schoultz, 1998 : 332) Par ailleurs, vers le milieu du Xx siècle, John Foster Dulles, Secrétaire d'État d'Eisenhower, aurait affirmé à propos des immigrants latino-américains aux États-Unis : "Tu les tapes un peu dans le dos et tu leur fais penser que tu les adores. " (ibid.). Bien que ces attitudes ne soient plus acceptables dans l'espace public, nous constatons avec l'analyse des enquêtes d'opinion publique que les immigrants latinos demeurent peu désirés aux USA. Par ailleurs, il importe de noter en vue de notre objet de recherche sur les Latinos d'origine mexicaine, que, selon ces enquêtes d'opinion sur l'acceptation des immigrants latino-américains, les Mexicains prendraient la place des immigrants les moins désirés.

La plupart des Latino-américains qui entrent dans le pays en tant qu'immigrants se soumettent involontairement à un processus de " racisation » et $d$ '" ethnicisation » qui imprègne leurs expériences et influence leurs revendications identitaires. Le phénomène de la racisation se produit dans la vie quotidienne à partir de leurs caractères phénotypiques (couleur de peaux, cheveux, physionomie). Le phénomène d'ethnicisation se produit tout d'abord par les désignations ethniques, comme celles d' "Hispanique » et de «Latino », retenues par le Bureau du recensement. L'interprétation des désignations ethniques et raciales du Bureau du recensement est due au système de classifications qui exige des citoyens et des visiteurs aux États-Unis de cocher « son groupe ethnique » et de signaler son appartenance « raciale» par choix subjectif. En effet, le gouvernement américain demande à ces citoyens et aux immigrants de " choisir " une catégorie ethnique et raciale. Les catégories sur les documents officiels d'immigration sont les suivantes : Blanc/Cauca- 
sien, Noir/Africain-Américain, Hispanique/Latino, Asiatique, Indien d'Amérique ou d'Alaska, d'Hawaï, parmi d'autres. Alors que la désignation "Hispanique " fut instaurée dès les années 1970, la catégorie "Latino » eut à cette époque une connotation intellectuelle et militante. Cette dernière, d'abord une auto-désignation, fut par la suite récupérée par le Bureau du recensement et désigne désormais la catégorie double d'« Hispanique/Latino »(Cohen, 2004 :35). Néanmoins, les connotations racisantes de ces deux désignations persistent à présent dans les discours des Latinos.

La catégorie Hispanic (hispanique) est devenue une catégorie " ethnique » aux États-Unis pour désigner tout individu ayant des origines dans un ou plusieurs pays de langue espagnole, sans distinguer les expériences diverses des personnes issues de milieux économiques et sociaux variés, d'histoires diverses et d'origines multiples (Oboler, 1995 : 1). Ce label fait partie des catégories non raciales et ethniques « officielles ». Cette catégorie est utilisée dans le recensement de la population, sur les documents de demande de visa pour entrer aux États-Unis, sur le formulaire de la déclaration d'impôts et sur les bulletins de vote. Bien qu'à présent le gouvernement définisse les Latinos en termes ethniques «non raciaux ", cette désignation porte des connotations raciales. Contrairement aux pays européens où le concept de "race » demeure peu utilisé (en raison d'une connotation biologique trop marquée), aux États-Unis, le concept de « race » a été redéfini comme une simple «identification sociale et culturelle». Néanmoins, pour beaucoup de Latinos, la classification « ethnique » semble souligner la stigmatisation des préjugés raciaux qui demeurent particulièrement présents aux États-Unis, et signale par là une certaine continuité avec un passé colonial douloureux (Alcoff, 2000; Oboler, 1995).

En revanche, le terme Latino est une catégorie plus récente, créée par les intellectuels et les militants pendant les mouvements identitaires des années 1970. C'est une catégorie inventée, vraisemblablement sans connotation raciale ou ethnique. La catégorie Latino décrit la partie de la population américaine qui rattache ses origines aux pays de l'Amérique espagnole, soit les Caraïbes et l'Amérique latine. Ainsi, le « Latino » est par définition blanc, noir, indigène, avec ou sans métissage. Alors que la catégorie d'Hispanique fut largement critiquée par la communauté Latina pour son effet homogénéisant visant à effacer la diversité ethnique, culturelle, sociale, religieuse, et linguistique, la catégorie Latino apparaît dans les mouvements identitaires et les politiques culturelles des immigrants et des descendants des Latino-américains dans les années 1970 comme une catégorie intellectuelle et militante. La désignation de Latino est connue comme une désignation des grassroots, elle est préférée par de nombreux Latinos. Pourtant, cette catégorie peut engendrer des effets racisants car, comme l'affirment Grosfoguel et Georas, même les personnes aux cheveux blancs et aux yeux bleus entrent dans le « labyrinthe de l'Altérité raciale » du moment où elles s'identifie comme Portoricaines (1996, cité dans Alcoff, 2000 :37). Néanmoins, on retiendra que la catégorie Latino semble correspondre à une auto-identification culturelle et politique. 
Par ailleurs, les catégories « Hispanique » et « Latino » sont parfois appréhendées comme des ethnonymes qui signaleraient un héritage ethnique et culturel désignant les personnes issues de l'Amérique latine (Latino) ainsi qu'un lien aux pays de langue espagnole (Hispanique). Or, il faut bien noter que les personnes issues de l'immigration des pays latino-américains ne parlent pas toutes l'espagnol et ne partagent pas un même héritage " ethnique » ou culturel. Ainsi, ces catégories portent d'abord l'inscription des discours de l'élite, qui définissent où et comment les populations issues de l'immigration sont incluses (ou non) comme des citoyens dans la société dominante et appartenant à la nation (Ong, $1996: 174)$.

De nos jours, la catégorie double « Hispaniques/Latinos » apparaît dans les statistiques du Bureau de recensement comme une catégorie ethnique «non raciale » formant une « minorité ethnique » et, depuis 2000, hispaniques/latinos peuvent choisir d'appartenir à un groupe racial quelconque (blanc, noir, race mixte etc.). Cette auto-désignation raciale a " quelque chose de subjectif ", mais elle peut avoir des effets concrets, dans l'emploi par exemple (Cohen, 2004 :34). C'est à partir des phénomènes de racisation que les Latinos « sentent » leurs différences avec les Blancs de sorte que nombre d'entre eux préfèrent choisir une identité ethnique blanche (Alcoff, 2000 :33). Or, la catégorie "Hispaniques/ Latinos " semble en fait fonctionner comme une catégorie " raciale ». Pour reprendre une phrase de Linda Alcoff : "On parle de nous en termes de culture, mais les gens pensent en termes de race. » (2000:37) I1 n'est donc guère surprenant que beaucoup de personnes «Latinos » dénoncent les catégories ethniques mises en avant par le Bureau de recensement, car, selon elles, " ethnique » résonne non seulement avec " pas blanc » (non-white), mais connote, par ailleurs, des attributs stéréotypés et péjoratifs d'une identité "pas blanche » (Alcoff, 2000 :37). Pour ces raisons, la désignation "Hispanique/Latino » et l'adjectif « ethnique » demeurent des termes problématiques pour beaucoup de Latinos. Une alternative nous est proposée par Alcoff (2000 :39). Elle souligne qu'il vaudrait mieux accepter « les réalités de la racisation, sans succomber à un certain fatalisme » ni essentialisme. Si les Hispaniques/Latinos sont une «population racisée » (Gracia \& Greiff, 2000 :3) par les politiques étatiques, nous nous essayerons de comprendre comment les stratégies locales visent à dépasser les catégories racisantes pour se transformer en des catégories d'appartenance culturelle.

Les immigrants latino-américains et les Latinos des États-Unis se trouvent sans cesse en train de négocier leur appartenance à un groupe linguistique ou culturel, à un espace de droit et à une communauté (Silvestrini, 1997; Rosaldo \& Flores, 1997). Ces revendications s'inscrivent dans des rapports de force inégaux dans lesquels s'insèrent les nouvelles politiques culturelles des Latinos et des immigrants latinoaméricains depuis les années 1990. En grande partie, les mobilisations récentes des Latinos plaident pour une " citoyenneté culturelle", qui permettrait d'améliorer les politiques de l'immigration et la reconnaissance de l'apport économique et culturel des immigrants latino-américains « sans papiers » qui viennent travailler aux États-Unis. 


\section{L'exemple des Latinos de l'Oregon}

Peu importe si les Latino-américains entrent aux États-Unis par voie légale (avec un visa de travail), par voie illégale (en traversant la frontière comme des "trempés ", mojados), qu'ils arrivent pour des raisons politiques ou économiques, la première expérience commune est celle de l'univers du travail. En effet, ce dernier figure comme un principe unificateur et un emblème de leurs expériences communes et de leurs souffrances partagées. Il faut rappeler que les immigrants latino-américains sont généralement associés au travail agricole même s'ils contribuent considérablement à la construction des voies ferrées, à l'industrie automobile et aux mines. Or, les générations ultérieures s'installent davantage en ville et changent d'occupation, engendrant ainsi une stratification sociale hétérogène. L'exemple des Latinos de l'Oregon abordé par la suite tient compte de l'expérience commune du travail et de l'hétérogénéité de leur communauté. Des extraits de récits des immigrants latino-américains et des récits des personnes qui habitent en ville et appartiennent à la génération des citoyens américains « Latinos » (des étudiants qui se sont engagés pour la cause des ouvriers agricoles dans l'Oregon) serviront à analyser les revendications de droits et d'une appartenance à une communauté latina des États-Unis.

La recherche s'est déroulée en partenariat avec le syndicat des ouvriers agricoles et immigrants latinos dans l'Oregon, Pineros et Campesinos del Nordeste (PCUN) sous la direction de l'anthropologue Lynn Stephen et avec la participation de plusieurs étudiants entre 1999 et 2001 (cf. Stephen, 2003) $^{31}$. Le syndicat PCUN fournit une assistance juridique pour aider à régulariser la situation des ouvriers sans papiers, et offre un conseil juridique aux ouvriers et à leurs familles. Les membres du PCUN, souvent eux-mêmes des anciens ouvriers agricoles sans papiers, surveillent, dans la mesure du possible, les conditions de travail dans les champs et vérifient les conditions de vie des labor camps (logement pour les ouvriers agricoles), souvent déplorables.

Lors des entretiens, les interviewés nous ont fait part de leurs expériences migratoires - par exemple, comment et combien de fois, ils ont traversé la frontière, par quelles voies, ainsi que les moments d'angoisse, de peur, ou de joie -, de leurs expériences et leurs souffrances dans les champs et dans les usines où ils travaillaient pour la plupart comme des ouvriers "illégaux » et "sans papiers ${ }^{1}$, puis de leurs difficultés et de leurs souffrances pour faire venir leur famille, pour obtenir des papiers. Ils ont aussi dit comment leur situation a changé - si elle a changé - au moment de l'obtention de ces papiers de résidence; enfin, ils ont parlé de leurs souhaits pour l'avenir de leurs enfants.

\footnotetext{
${ }^{1}$ Il faut préciser que nous avons mené la moitié de nos entretiens avec des personnes que l'on qualifierait selon la loi de « sans papiers » alors qu'ils paient des impôts aux États-Unis. En d'autres termes, le terme «sans papiers » est imprécis pour décrire leur situation.
} 


\section{LES EXPÉRIENCES DES OUVRIERS AGRICOLES D'ORIGINE MEXICAINE}

\section{DANS L'OREGON}

Les Mexicains ont une histoire singulière qui les distingue des autres Latinos et leur donne un statut particulier aux États-Unis. Si les relations entre les États-Unis et l'Amérique latine sont, certes, marquées par la globalisation, les relations entre les États-Unis et le Mexique ont, depuis toujours, été d'abord des rapports économiques et politiques. Le lien entre les États-Unis et le Mexique est marqué par l'inégalité de pouvoir; les ÉtatsUnis sont le puissant voisin qui peut se permettre de poser les conditions de leur entente. De manière très concrète, les institutions étatiques étatsuniennes déterminent qui peut traverser la frontière pour venir travailler aux États-Unis, et quand les ouvriers doivent retourner chez eux (généralement après la fin des récoltes, soi-disant, après six à huit mois de travail).

Parmi nos interlocuteurs mexicains dans l'Oregon, plusieurs hommes étaient arrivés pour la première fois dans les années 1950 comme des ouvriers migrants avec un visa de travail temporaire. Ils faisaient partie de la première vague des braceros. ${ }^{2}$

Le témoignage de Sofia, une femme d'origine mixtèque qui travaille sur les champs de l'Oregon, illustre les conditions difficiles de travail : « J'ai quitté le Mexique en 1994 pour retrouver mon mari qui travaille ici depuis quelques années, et j'ai commencé à travailler dans les champs de fraises. Je suis partie tôt le matin tous les jours à quatre heures pour commencer à travailler, et il fallait tout de suite trouver un bon endroit où il y avait beaucoup de fruits, car on nous payait au kilo. À l'époque, ils nous payaient seize cents pour 500 grammes de fraises. On ne s'arrêtait pas pour manger, parce qu'on ne pouvait perdre une demi-heure. En général, le travail durait jusqu'à 14 ou 15 heures. La moitié des ouvriers, c'est des femmes. Pour celles d'entre nous qui avions des enfants, c'était plus dur car on n'avait plus le droit d'amener nos enfants dans les champs. Il fallait donc payer une baby-sitter. Le travail est très dur car il faut rester accroupi toute la journée et cela fait mal au dos. "

Il y a environ 100000 ouvriers agricoles qui travaillent dans les champs de l'Oregon, dont 98 \% sont des Latinos, des immigrants latinoaméricains des résidents ou des citoyens, principalement d'origine mexicaine et d'Amérique centrale. Un grand nombre d'entre eux réside dans l'Oregon toute l'année afin de suivre le cycle des récoltes. En général, les ouvriers (hommes ou femmes) viennent d'abord seuls, sans leur famille. Faute de connaissances en anglais et faute de contacts dans la région, beau-

\footnotetext{
${ }^{2}$ Le Programme Bracero (1942-1964) est le premier accord entre les États-Unis et le gouvernement mexicain qui a permis le séjour temporaire de Mexicains en tant qu'« ouvriers migrants» (migrant worker). Ce programme a créé une immigration temporaire «légale » sans pour autant offrir des récompenses pour leur investissement dans l'économie américaine. Le Programme Bracero a par la suite créé des réseaux de mobilité d'ouvriers agricoles, pour fournir la main d'œuvre des champs de Californie et d'Oregon, qui vient principalement du Mexique et, depuis quelques années, du Guatemala.
} 
coup d'entre eux habitent d'abord dans des logements (des baraques provisoires ou de vielles maisons, souvent mal équipées pour recevoir un grand nombre de personnes) mis à disposition pour location par les farmers. Ensuite, après avoir fini la récolte en Californie, une grande partie d'entre eux poursuit son chemin à la recherche de travail en suivant les récoltes vers le Nord (Oregon, Washington), et quelques-uns redescendent au Texas avant de repartir au Mexique. Tout comme les Latinos, les ouvriers agricoles forment un groupe très hétérogène : il y a des ouvriers qui sont là depuis quarante ans et ont parfois obtenu une carte de résident, voire la citoyenneté. D'autres ont fait venir leur femme, et leurs enfants, nés sur le sol américain sont des ressortissants américains, alors que les parents attendant toujours leurs papiers et craignent l'expulsion à tout moment. D'autres encore ont obtenu des papiers avant de faire venir leurs familles et doivent cacher femme et enfants. Il est d'ailleurs assez commun que les Latinos avec papiers, ainsi que les « citoyens naturalisés " (naturalized citizens, ceux qui sont devenus des citoyens américains par voie administrative) continuent à travailler dans les champs car ils ont du mal à trouver un autre emploi.

En 1986, les États-Unis ont attribué des cartes de résidence légale à 2,8 millions d'immigrants à la suite de l'Immigration Reform and Control Act (IRCA), et plus d'un million d'ouvriers agricoles « sans papiers » ont été « légalisés » grâce à un programme spécialisé, le Special Agricultural Worker Program (SAw). Selon les estimations du Service de l'immigration et de la naturalisation (INS), il y avait, à la fin des années 1990, entre six et neuf millions d'ouvriers sans papiers, et d'autres entraient dans le pays au rythme de deux cent mille à trois cent mille par an (Stephen, 2003). La stigmatisation et les pratiques d'exclusion des Latinos qui sont des ouvriers agricoles reste actuelle dans la vie de ces personnes et ne cesse pas nécessairement quand ils obtiennent des papiers. L'exemple de Lorenzo, un des organisateurs du PCUN qui a été ouvrier agricole pendant trente ans, partage ses expériences dans les champs et revendique un espace de droits pour les ouvriers mexicains : « Je m'appelle Lorenzo et je suis Indien mixtèque. Je suis né à Oaxaca au Mexique dans les années cinquante. Dans les années soixante-dix, je suis arrivé en Californie pour travailler dans les champs. La discrimination et l'exploitation des ouvriers étaient très graves. J'ai vu beaucoup de choses, par exemple les patrons qui battaient les ouvriers. Au lieu de nous parler, ils nous bousculaient violemment. Avec le PCUN, nous souhaitons que les mayordomos [ceux qui surveillent les ouvriers sur les champs] traitent les ouvriers avec respect, qu'ils leur autorisent une pause, d'aller pisser, et qu'ils aient le droit de parler entre eux. Souvent c'est difficile car ce sont les farmers qui ont le pouvoir et les ouvriers n'en ont pas. Les ouvriers ont besoin d'avoir des droits comme d'autres personnes. »

\section{LA MOBILISATION DES ÉTUDIANTS LATINOS}

Les étudiants qui ont participé à ce projet ont retrouvé les expériences de leurs parents et de leurs grands-parents. Ils ont évoqué l'im- 
portance de rendre publiques les souffrances d'une grande partie de la population latina qui continue à vivre dans la clandestinité, mais ils contribuent pourtant au chiffre d'affaires des farmers et de l'État, ainsi que du pays. Claudia, une étudiante, qui était à l'époque militante dans l'organisation des étudiants "Chicano » (Latino d'origine mexicaine) à l'Université d'Oregon, est née en Californie de parents mexicains qui étaient des ouvriers dans les champs. Elle fait partie de la première génération de citoyens états-uniens d'origine latina. Ses parents avaient réussi à légaliser leur situation dans les années 1970, avant sa naissance. Dans son discours, Claudia s'identifie aux hommes et femmes qui travaillent dans les champs. Son empathie est liée à sa propre histoire et à celle de ses parents, et aussi celle de ses proches, car un ami était un " sans papiers ", autrement dit, une personne vivant dans un espace « sans droits" (civiques et culturels) mais avec des devoirs (par exemple celui de payer des impôts) : "Je suis une femme Latina dans ce pays et je comprends la situation des personnes qui sont arrivées récemment, parce que ma famille est passée par là. Il s'agit d'une injustice de faire venir des personnes et des les renvoyer quand ils n'en ont plus besoin. Comment sont-ils censés vivre pendant des mois et des mois loin de leurs familles? Comment font les enfants pour aller à l'école s'ils doivent déménager tous les mois dans un autre État. Et puis, même nous, les Latinos qui sommes des citoyens, on nous traite parfois comme des ordures. J'ai de la chance parce que j'ai la peau plutôt blanche et donc les gens ne savent pas que je suis issue d'un milieu modeste, mais mes amis qui ont la peau plus foncée souffrent plus que moi. J'ai participé à ce projet pour mieux comprendre la situation de notre communauté latina et pour éduquer les autres personnes, car les Latinos, nous avons notre culture et nos communautés, nous faisons partie de la société, de l'économie et nous demandons d'avoir les droits comme les autres. »

Ce témoignage souligne plusieurs points importants. Tout d'abord, l'expérience commune des conditions difficiles du travail - celle-ci est étroitement liée à des expériences de racisation qui semblent toucher tantôt les immigrants récents, tantôt les citoyens américains Latinos qui ont une apparence différente de celle des « Blancs ». Puis, il souligne l'importance de la communauté latina et signale un fonds culturel commun. Enfin, ce récit suggère la création d'une identité " pan-latina " quand Clausia revendique " notre culture " et " notre communauté latina " et réitère ainsi les revendications des ouvriers agricoles à un espace de droits culturels et civiques pour tous les Latinos, autrement dit, pour tous ceux qui participent à la société américaine par leur travail, par leurs impôts, et par leur apport social.

\section{ENJEUX ÉCONOMIQUES, DÉMOGRAPHIE, ET DROITS}

Nos réflexions sur les Latinos des États-Unis et les récits des ouvriers agricoles d'origine mexicaine et des étudiants latinos invite à repenser le modèle universaliste de la citoyenneté qui préconise l'individu comme 
un sujet de droits. La population latina dans l'Oregon ne cesse d'augmenter ${ }^{3}$. Les causes de l'immigration sont à chercher dans la nécessité d'une main d'œuvre bon marché pour supporter l'économie agricole. Les ouvriers latinos répondent à ce besoin sans bénéficier de droits culturels et politiques. Aux États-Unis, le discours d'une « citoyenneté universelle » et l'idéal d'une société multiculturelle restent une simple rhétorique sans pour autant reconnaître les contributions économiques, sociales, culturelles et politiques des immigrants. À l'heure actuelle, la situation des ouvriers "sans papiers " qui traversent la frontière de manière périlleuse est de plus en plus dénoncée par les Latinos dans l'espace public, par exemple par des rassemblements contre la construction d'un mur le long de frontière avec le Mexique. Cette situation précaire et les critiques des pratiques de racisation des immigrants latinoaméricains font avancer les revendications d'une identité pan-latina en faveur d'une communauté où tous les membres bénéficieraient de droits culturels et collectifs.

\section{LA CONSTRUCTION D'UNE CITOYENNETÉ CULTURELLE LATINA}

La littérature sur la citoyenneté et la nationalité a longtemps ignoré les expériences subjectives et contradictoires. Suivant les idéaux des philosophes des Lumières, la rhétorique de la constitution des États-Unis proclame une citoyenneté " universelle » pour tous les citoyens pourvu qu'ils partagent la même langue et la même culture. Les études se sont d'abord intéressées aux tensions entre le sujet souverain et la solidarité envers un État-nation. On peut distinguer deux approches principales : d'une part, les études privilégiant les aspects légaux et politiques en faveur d'une citoyenneté universelle, d'autre part, les études qui ont souligné les contradictions entre la rhétorique d'une citoyenneté démocratique universelle et les inégalités créées par l'économie marchande et ses effets marginalisants. William McNeill(1986) souligne que l'idéal d'une nation homogène est illusoire et demeure une idéologie jamais achevée dans la pratique. Une nationalité, généralement entendue comme une catégorie politique, peut comprendre plusieurs ethnicités en pratique. Ces deux approches ont privilégié les critères " universaux » de la citoyenneté et, par extension, défenseurs d'une nationalité supposée homogène, sans tenir compte des positions des sujets (subject positions) au sein d'un ÉtatNation, en particulier les sujets issus d'une immigration qui augmente avec la globalisation.

Suivant les travaux de Stuart Hall et David Held (1990), les précurseurs de l'étude des «nouvelles politiques de la citoyenneté ", Renato

\footnotetext{
${ }^{3}$ Selon les enquêtes nationales, il y avait 112700 personnes de langue espagnole dans l'Oregon en 1990. En 2000, le chiffre a atteint 275300 (soit + 114,4\%). De ces hispanophones, environ $80 \%$, étaient d'origine mexicaine. On estime que dans les dix ans qui viennent, la population des Latinos dans l'Oregon doublera pour atteindre $20 \%$ de la population totale de l'État (Stephen, 2003).
} 
Rosaldo (1994) fut un des premiers à définir la « citoyenneté culturelle » comme un processus de revendication d'appartenance et de citoyenneté, à part entière, des personnes marginalisées aux États-Unis. Les Latinos commencent à y revendiquer des droits collectifs civiques et culturels en réponse aux désignations ethniques et aux processus de racisation et d'ethnicisation dans leur vie quotidienne, ce qui montre la nécessité de développer un nouveau modèle pour définir la citoyenneté comme une citoyenneté culturelle.

William V. Flores et Rina Benmayor (1997) proposent de reconnaître les immigrants mexicains et latinos comme des sujets politiques légitimes réclamant des droits collectifs culturels pour leurs enfants et pour leurs familles à hauteur de leur contribution et de leur participation à la vie économique et sociale du pays (Flores \& Benmayor, 1997 : 15). Lynn Stephen (2003) prolonge cette réflexion lorsqu'elle pense la citoyenneté culturelle des Latinos comme l'ouverture d'un espace de droits civiques, garante du respect de la personne et de la reconnaissance de ses droits culturels. Ainsi, la notion de « citoyenneté culturelle » constituet-elle une alternative à la " citoyenneté universelle ", en ce qu'elle permet aux ouvriers sans papiers, au statut officiel d'" étranger illégal ", de faire reconnaître leur contribution à la société américaine et d'affirmer leur ancrage dans des communautés transnationales. En somme, il s'agit d'un processus de subjectivation, dans le sens d'Ong (1996), qui se produit entre les politiques de citoyenneté des États-Unis, déterminant l'appartenance à la nation et au territoire, et les expériences subjectives des Latinos

\section{Références bibliographiques}

Alcoff L. M., "Is Latina/o Identity a Racial Identity? " in Gracia J.J.E. \& GreIFF P. DE (dir.), Hispanics/Latinos in the United States. Ethnicity, Race, and Rights, New York \& Londres, Routledge, 2000, p. 2344.

Cohen J., "Logiques socio-politiques de la "latinisation" des ÉtatsUnis » in Cohen J. \& Tréguer A. (dir.) Les Latinos des USA, Paris, IHeALCREDAL Éditions, 2004, p. 31-47.

Cohen J. \& Tréguer A., "Les États-Unis dans les Amériques : pays en voie de "latinisation"? " in COHEN J. \& Tréguer A. (dir.) Les Latinos des USA, Paris, IHEAL-CREDAl Éditions, 2004, p. 9-14.

Delgado R. \& Stefancic J., "Introduction " in Delgado R. \& Stefancic J. (dir.), The Latino Condition : A Critical Reader, New York \& Londres, Routledge, 1998, p. XVII-XIX.

Gracia J.J.E. \& GREIFF P. DE, « Hispanic/Latino Ethnicity, Race and Rights » in Gracia J.J.E. \& GreifF P. DE (dir.), Hispanics/Latinos in the United States. Ethnicity, Race, and Rights, New York \& Londres, Routledge, 2000, p. 1-20.

Hall S. \& Held D., "Citizens and Citizenship " in Hall S. \& Jaceues M. (dir.), New Times: The Changing Face of Politics in the 1990s, Londres, Verso, 1990, p. 173-188. 
Oboler S., Ethnic Labels, Latino Lives. Identity and the Politics of (Re)Presentation in the United States, Minneapolis, U. of Minnesota Press, 1995.

Ong A., "Cultural Citizenship as Subject-Making : Immigrants Negotiate Racial and Cultural Boundaries in the United States ", Current Anthropology, 377(5), 1996, p. 737-762.

Rosaldo R., "Cultural Citizenship in San Jose, California », Polar 17, 1994, p. 57-63.

- "Cultural Citizenship, Inequality, and Multiculturalism " in Flores W.V. \& Benmayor R. (dir.), Latino Cultural Citizenship : Claiming identity, Space, and Rights, Boston, Beacon Press 1997, p. 27-38.

— \& Flores W.V., "Identity, Conflict, and Evolving Latino Communities : Cultural Citizenship in San Jose, California » in Flores W.V. \& Benmayor R. (dir.), Latino Cultural Citizenship: Claiming identity, Space, and Rights, Boston, Beacon Press 1997, p. 57-96.

Schoultz Lars Van Trier, Beneath the United States : A History of U.S. Policy Toward Latin America, Boston, Harvard U. P. 1998.

Silvestrini B.G., "World We Enter When Claiming Rights' : Latinos and Their Quest for Culture » in FLORES W.V. \& BENMAYOR R. (dir.), Latino Cultural Citizenship : Claiming identity, Space, and Rights, Boston, Beacon Press 1997, p. 39-53.

Stephen L., "Cultural Citizenship and Labor Rights for Oregon Farmworkers : The Case of Pineros y Campesinos Unidos del Noreste (PCUN) », Human Organization, 62(1), 2003, p. 27-38. 